\title{
Des objets intermédiaires pour aider aux coordinations entre éleveurs et laiterie. L'exemple d'une coopérative laitière caprine du sud de la France
}

\author{
M. Napoléone ${ }^{1 *}$
}

Mots-clés

Caprin, collecte du lait, coopérative laitière, saisonnalité, éleveurs, recherche opérationnelle, zone méditerranéenne

Accepted: 24 April 2019

Published: 12 July 2019

DOI: 10.19182/remvt.31746

\section{Résumé}

La gestion de la saisonnalité de la production laitière est une difficulté pour les entreprises de collecte et de transformation. Nous avons exploré ici l'intérêt de valoriser des complémentarités entre des systèmes de production présents sur un bassin de collecte. La mise en place d'un dispositif de coordination a été proposée pour identifier ces complémentarités au regard d'un enjeu négocié entre les éleveurs et la laiterie. Il a été conçu comme un itinéraire : a) la formalisation des activités élémentaires, b) l'analyse de leurs interactions, c) la formulation d'un diagnostic concerté, et d) I'identification de pistes d'action. A chaque étape ont été proposées des représentations graphiques construites à partir des pratiques des acteurs et des objets sur lesquels elles portaient. Ces représentations ont été utilisées comme des objets intermédiaires. A partir d'un projet de rechercheaction conduit avec une petite coopérative laitière du sud de la France, nous avons montré que les représentations des livraisons ou de la collecte pouvaient être utilisées comme des objets intermédiaires permettant de rendre lisibles les modes de conduites des troupeaux ou la stratégie commerciale de la coopérative, facilitant l'analyse des interactions entre ces activités et l'élaboration d'un projet commun. En conclusion, des représentations simples et pragmatiques se sont avérées être des outils efficaces et intéressants à mobiliser dans le cadre d'actions collectives impliquant une coordination entre éleveurs et coopératives.

- Comment citer cet article : Napoléone M., 2019. Intermediate objects to help coordination between breeders and dairies. Example of a goat dairy cooperative in Southern France. Rev. Elev. Med. Vet. Pays Trop., 72 (2): 55-63, doi: 10.19182/remvt.31746

\section{INTRODUCTION}

La gestion de la saisonnalité de la production laitière est une difficulté pour les éleveurs comme pour les gestionnaires de laiterie. En effet, les livraisons de lait sont saisonnées, alors que la demande en produits laitiers l'est beaucoup moins. De fait, à certaines saisons, les laiteries manquent de lait, alors qu'à d'autres elles font face à un afflux de lait à transformer et à vendre. L'ajustement amont et aval est un enjeu pour les laiteries, quel que soit le type de lait transformé. Il est plus difficile à réaliser en production caprine, du fait de la saisonnalité marquée de la reproduction caprine dans le contexte français métropolitain. Les

1. INRA SAD, UMR Selmet, 2 place Pierre Viala, F-34060 Montpellier, France. SELMET, Univ Montpellier, INRA, CIRAD, Montpellier, France. Email : martine.napoleone@inra.fr

* Ces résultats ont été en partie présentés lors de l'atelier INRA-CIRAD Systèmes d'information et outils de pilotage du secteur élevage dans les pays du Sud. Postures et méthodes (coord. Duteurtre G., Bonnet P., Dutilly C., Napoléone M., Touré I.), Montpellier, France, 11-13 juil. 2011 gestionnaires des laiteries disposent de divers outils pour agir sur la répartition de la collecte : établir une grille de prix du lait en faveur du lait d'hiver, inciter les éleveurs à produire en basse saison, congeler au printemps une partie du caillé pour en reporter sur l'automne la transformation, et fabriquer des produits de report. Cependant, malgré les mesures qui peuvent être prises, la saisonnalité de la collecte reste une difficulté récurrente dans la plupart des entreprises de transformation laitière. Cette difficulté est exacerbée quand l'entreprise est une coopérative statutairement redevable de transformer $100 \%$ du lait produit par ses adhérents.

Dans cet article nous avons examiné les actions pouvant atténuer la saisonnalité de la collecte laitière d'une coopérative. La diversité des systèmes d'élevage présents sur le bassin de collecte peut-elle devenir un moyen d'action pour réguler la collecte au cours de l'année ? Comment accompagner les acteurs (éleveurs et gestionnaires) dans l'identification de ces complémentarités et des marges de manœuvre envisageables ?

Le choix s'est porté sur l'intérêt de mettre en place un dispositif sur le temps long permettant aux acteurs de cheminer de la formalisation des activités présentes à l'analyse de leurs interactions, à la formalisation d'un diagnostic concerté et, enfin, à l'identification 
de pistes d'action. Nous avons émis l'hypothèse que des représentations simples et pragmatiques, construites à partir des pratiques des acteurs, facilitaient la coordination en aidant à la construction de sens et à l'identification de pistes d'action.

Pour illustrer cette démarche, une recherche-action a été menée avec des éleveurs et des gestionnaires d'une petite coopérative laitière caprine du sud de la France. Les représentations graphiques construites avec les acteurs sont présentées et leurs rôles aux diverses étapes du dispositif de recherche-action sont analysés. Cette démarche partageait plusieurs principes avec celle de la modélisation d'accompagnement Commod (Daré et al., 2009 ; Bousquet et al., 2002 ; Etienne, 2011). Le dispositif visait à organiser des itérations successives entre l'action (ou modélisation de l'action) et l'analyse (Napoléone et al., 2008). Pour faciliter la mise en situation et l'échange de points de vue, des représentations graphiques coconstruites avec les acteurs ont été utilisées. Toutefois, à la différence des démarches Commod, nous avons construit des représentations au plus près des pratiques des acteurs, en lien direct avec leurs activités de tous les jours, pour faciliter la mise en situation et l'appropriation suite à l'exercice de modélisation.

\section{- PROJET DE RECHERCHE-ACTION AVEC UNE PETITE COOPERATIVE}

\section{Emergence du projet et négociation de la question à traiter}

La mise en place de l'appellation d'origine contrôlée (AOC) Pélardon (fromage de chèvre originaire des Cévennes) en 2000 a interdit l'utilisation de caillé congelé pour la transformation. Ceci a supprimé le principal moyen de report utilisé par la coopérative laitière partenaire du projet de recherche-action. Dans le cas de ce fromage à faible durée d'affinage, l'interdiction de report congelé a élargi la période creuse et a obligé la laiterie à gérer en temps réel l'ajustement entre la saisonnalité de la collecte et celle des ventes. Or, le signe officiel de qualité AOC permettrait à la coopérative Fromagerie des Cévennes, le plus gros producteur de Pélardon de la région, de sortir du marché régional saturé et d'écouler ses produits sur des marchés éloignés de la zone d'origine, notamment par les circuits de la grande distribution nationale. Cependant, la demande en circuit long est peu saisonnée alors que la collecte de cette laiterie est trois fois plus importante au printemps qu'en automne. Les volumes collectés en automne limitent les quantités annuelles négociables auprès des circuits longs. Malgré une grille de prix mise en place depuis 10 ans et la diffusion de conseils aux producteurs pour dessaisonner la reproduction de leur troupeau, la collecte reste saisonnée (Napoléone, 2008).

Dans le cadre d'un appel d'offres INRA-Région (Programme sur et pour le développement régional [PSDR]) le directeur de la laiterie a proposé de construire un projet de recherche-action pour étudier des pistes pour agir sur la saisonnalité de la collecte de la coopérative. Il considérait que le problème principal était lié à de la résistance à l'innovation des éleveurs ou à des difficultés techniques freinant l'adoption des pratiques nécessaires à la reproduction en contre-saison. Il a donc proposé à la recherche d'étudier la façon d'accompagner des changements de pratiques en élevage vers des systèmes dessaisonnés. Nous avons postulé qu'un changement de période de reproduction ne se réduisait pas à des difficultés techniques, qu'il engageait aussi des réorganisations en profondeur du système d'élevage, du travail, de l'utilisation des ressources et qu'il interrogeait la conception que les éleveurs avaient de leurs activités et de leur métier. Nous avons aussi postulé que la mise en avant d'un modèle technique en opposition aux autres n'était pas favorable à l'émergence d'une dynamique d'action collective au sein de la coopérative. Nous avons enfin fait l'hypothèse qu'il existait une diversité de systèmes d'élevage sur le bassin de collecte ayant leur saisonnalité propre et que cette diversité pouvait constituer une ressource pour agir sur l'ajustement entre la saisonnalité des livraisons et celle des ventes. En effet, une étude menée avec une coopérative caprine de 100 adhérents dans la Drôme (Napoléone, 2001) montre que la diffusion d'un modèle unique au sein d'une coopérative ne réduit pas le problème de saisonnalité de la collecte mais le déplace de l'automne vers la fin de l'été. Cette étude montre aussi qu'il existe une diversité de profils de production parmi les adhérents, étroitement liée aux pratiques de conduite du troupeau, donc à la diversité des systèmes d'élevage.

Dans le prolongement de cette étude, la coopération avec une petite coopérative de 27 adhérents a permis d'explorer l'intérêt d'utiliser des représentations graphiques factuelles pour accompagner les acteurs dans l'analyse de situation et la recherche de pistes d'action sur la saisonnalité via l'identification de complémentarités entre systèmes d'élevage. La question à traiter, le type de résultat visé et la posture de recherche ont été négociés avec le directeur de la coopérative et son conseil d'administration (CA). Il a été convenu de mettre en place un dispositif de recherche-action permettant de rendre lisibles, pour les éleveurs et pour les gestionnaires de la coopérative, étape après étape, les diverses activités en interaction (systèmes d'élevage et gestion de collecte), de faciliter la formulation d'un diagnostic de situation en commun et l'identification d'actions. Cette étape préliminaire a été indispensable pour la mise en œuvre du projet. Elle a permis aux partenaires (chercheurs et acteurs) de s'entendre sur la formulation de la question à traiter, sur la façon de la traiter à travers la mise en place d'un dispositif sur deux ans et sur la posture de recherche (animer en accompagnant pas à pas la construction d'une vision commune).

\section{Dispositif pour structurer le processus de conception innovante}

La collecte laitière pouvait être vue comme le résultat d'un ensemble de processus et de prises de décisions individuelles et collectives. Cependant les acteurs n'avaient pas une lisibilité de l'ensemble de ces processus en interaction. Les gestionnaires de la coopérative avaient une perception de la diversité à travers leurs indicateurs relatifs aux volumes livrés dans l'année ou à telle ou telle saison. Tous les éleveurs n'avaient pas non plus les mêmes objectifs, les mêmes façons de concevoir leurs activités, les mêmes facteurs de production, ni les mêmes modes de gestion de leur exploitation et de leur troupeau. Augmenter la lisibilité de ces processus était un préalable pour aider les acteurs à identifier des pistes d'action individuelles et collectives. Le concept d'action collective conjointe de Lorino et Nefussi (2007) était intéressant à mobiliser. Pour ces auteurs, faciliter la coordination d'acteurs en vue de la construction d'une action collective conduit à les aider à avoir une lisibilité de la situation (la diversité des systèmes d'élevage, les problématiques d'ajustement amont et aval), à formuler ensemble la question à traiter, et à identifier la contribution de chacun à l'élaboration et à la gestion de cette ressource commune (ici la collecte laitière).

Nous avons mis en place un dispositif sur deux ans (figure 1) (Napoléone et Chia, 2010). Il a été conçu comme un cheminement, en trois étapes : étape 1, aider chaque acteur à décrire et à formaliser ses activités et leurs évolutions ; étape 2, faciliter l'élaboration d'une vision commune des activités et de leurs interactions ; et étape 3, formuler un diagnostic en commun et identifier les pistes d'action. Chaque étape s'est appuyée sur la coconstruction de représentations graphiques, souvent calendaires, utilisées comme support pour faciliter l'expression des façons de faire et de voir de chaque acteur, et l'échange entre acteurs, ou pour faciliter les coordinations.

Nous avons distingué les « éleveurs » pour évoquer l'activité d'élevage dans l'exploitation et les « gestionnaires-coop » pour désigner celle de gestion de la collecte (directeur-salarié ou éleveurs-membres du CA). Le terme « acteurs » faisait référence à l'ensemble [éleveurs et gestionnaires-coop]. Les chercheurs ont géré l'avancée du dispositif et l'accompagnement des acteurs. 


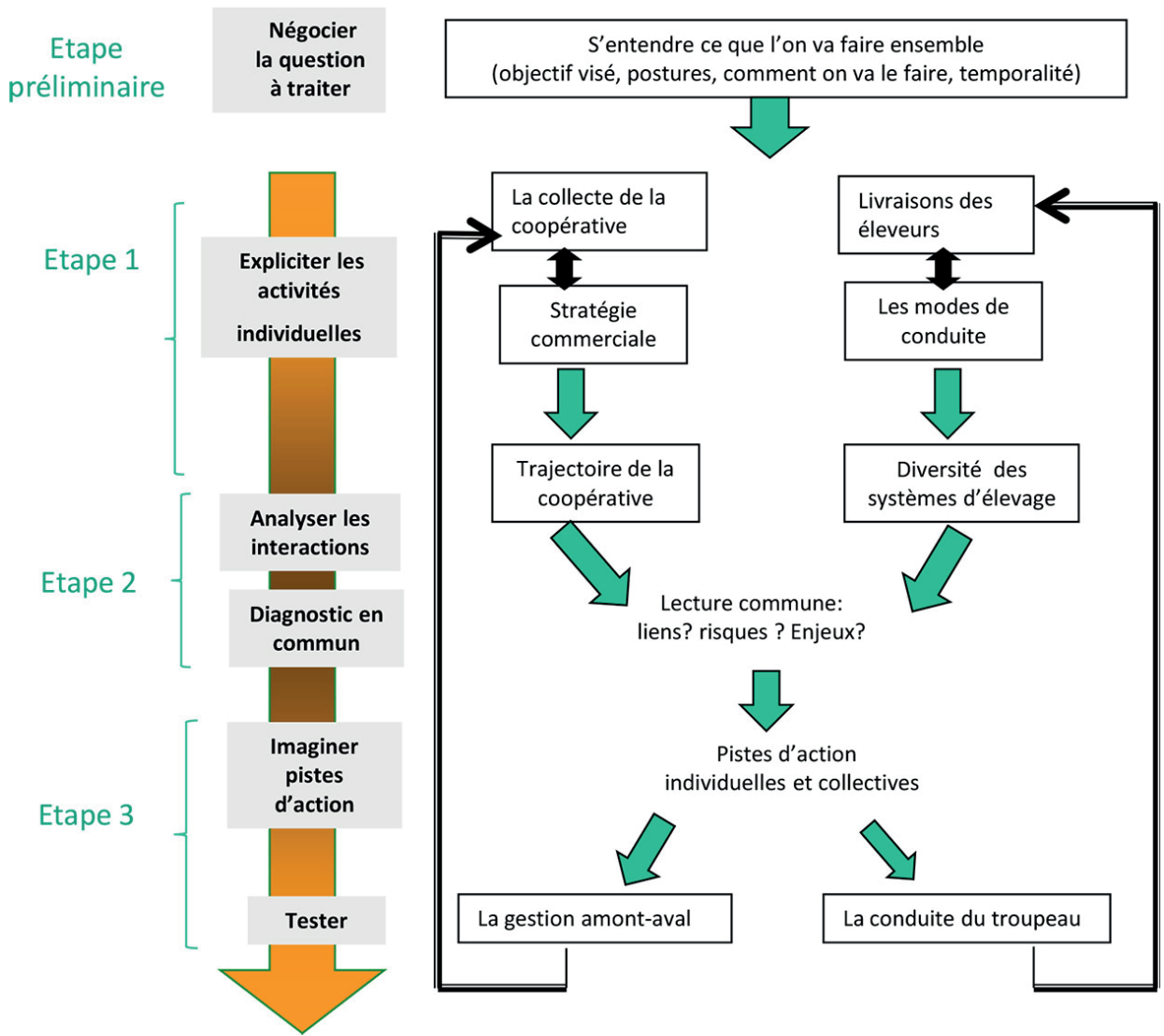

Figure 1 : étapes du dispositif de recherche-action visant à faciliter la coordination entre la coopérative laitière caprine cévenole et les éleveurs.

\section{ETAPES DU DISPOSITIF ET REPRESENTATIONS GRAPHIQUES MOBILISEES}

\section{Aider chaque acteur à décrire et formaliser ses activités et leurs évolutions (étape 1)}

Des entretiens compréhensifs individuels ont été réalisés avec chaque acteur (27 éleveurs et 4 gestionnaires-coop). L'objectif était de rendre lisibles leurs activités en mettant en évidence les manières de les conduire et en permettant à chaque acteur d'exprimer sa façon de les gérer, les difficultés rencontrées, les perspectives entrevues et les cohérences qui les sous-tendent (la stratégie et le mode de conduite du troupeau pour les éleveurs, la stratégie commerciale pour les gestionnaires). Chacun d'eux a décrit le déroulement de ses activités sur l'année et son évolution sur le temps long. Pour enclencher ces narrations, la courbe de livraison de l'éleveur ou l'évolution de la collecte (évolution de quantité annuelle collectée depuis 1974 et évolution des profils de collecte à partir des volumes mensuels) sur le temps long ont été utilisées comme objets intermédiaires (Vinck, 1999). Le lait livré par chaque éleveur correspondait au lait produit par le troupeau, indicateur quotidien à partir duquel l'éleveur appréciait la situation et ajustait ses pratiques (par exemple changements de parcs). Le gestionnaire de laiterie utilisait comme indicateur le lait collecté, somme des laits livrés par les coopérateurs, pour suivre l'évolution de l'approvisionnement et l'ajustement aux ventes. Le lait-troupeau ou le lait collecté étaient donc des repères mobilisés par les acteurs dans leurs activités quotidiennes. A la vue des courbes obtenues, nos interlocuteurs sont revenus dans l'action en décrivant le déroulement de leur activité sur une campagne complète (figure 2). Les éleveurs ont mis en relation l'évolution du lait livré avec leurs pratiques d'alimentation, de pâturage, de reproduction, le travail et les événements ayant ponctué la campagne. L'évolution de la collecte a renvoyé les gestionnaires à l'évolution des circuits commerciaux et des produits sur le temps long, aux difficultés rencontrées pendant la durée de leur activité, et aux incitations de la coopérative envers les éleveurs.
Les représentations graphiques qui ne schématisaient au départ que les livraisons ou la collecte se sont enrichies des pratiques, des entités sur lesquelles elles portaient, des temporalités dans lesquelles elles s'opéraient. Nous avons ainsi coconstruit, au fil de chaque entretien, de nouveaux graphiques, factuels et calendaires, représentant l'organisation sur l'année et l'évolution sur le long terme de chaque activité élémentaire (figure 3). Ils ont été un support de réflexion pour cheminer de la description de ce qui est fait à la caractérisation du mode d'organisation (entre autres, entités de gestion, identification de périodes, enchaînement entre périodes, leviers d'action), et aux cohérences sous-jacentes.

Dans le cas des éleveurs, un écrit a permis d'acter cette réflexion. Nous y avons consigné a) la représentation factuelle visualisant la courbe de livraison, et le calendrier de conduite du troupeau et d'utilisation du pâturage, et b) les appréciations de l'éleveur et la façon dont il exprimait sa stratégie de production. Cet écrit a permis une double validation par l'éleveur, d'une part, du cadre de représentation des systèmes d'élevage (une base temporelle pour représenter les liens entre les pratiques de conduite et la saisonnalité de la production de lait du troupeau) et, d'autre part, de la description de son système (stratégie, fonctionnement, diagnostic). La validation par chaque acteur de la façon de représenter et de rendre lisibles ses activités a eu un rôle essentiel dans le dispositif. Elle a permis de construire un sens partagé sur la façon de représenter et d'analyser les liens pratiques-production.

\section{Faciliter l'élaboration d'une vision commune des activités présentes et de leurs interactions (étape 2)}

\section{Augmenter l'interconnaissance des activités présentes}

- De l'évolution de la collecte à la trajectoire de la coopérative et à la formulation du problème de saisonnalité

Nous avons réuni les informations issues des entretiens individuels avec les quatre gestionnaires-coop dans une seule représentation de la 


\author{
Les relevés de livraison \\ Permettent de représenter la \\ courbe de lait troupeau
}

La courbe de lait du troupeau

Constitue un support à la narration par l'éleveur de sa conduite

\section{La représentation de la conduite} du troupeau

La narration de l'éleveur permet de représenter la conduite, ici le pâturage

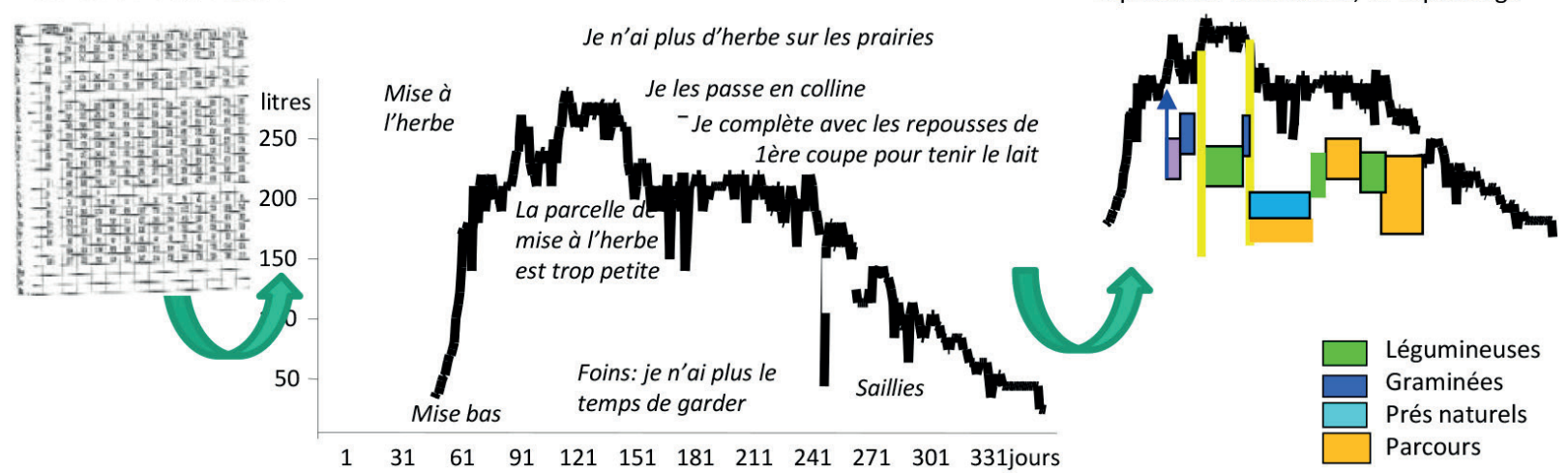

Figure 2 : de la courbe laitière du troupeau de chèvres d'un éleveur cévenol à la représentation de sa conduite du troupeau.

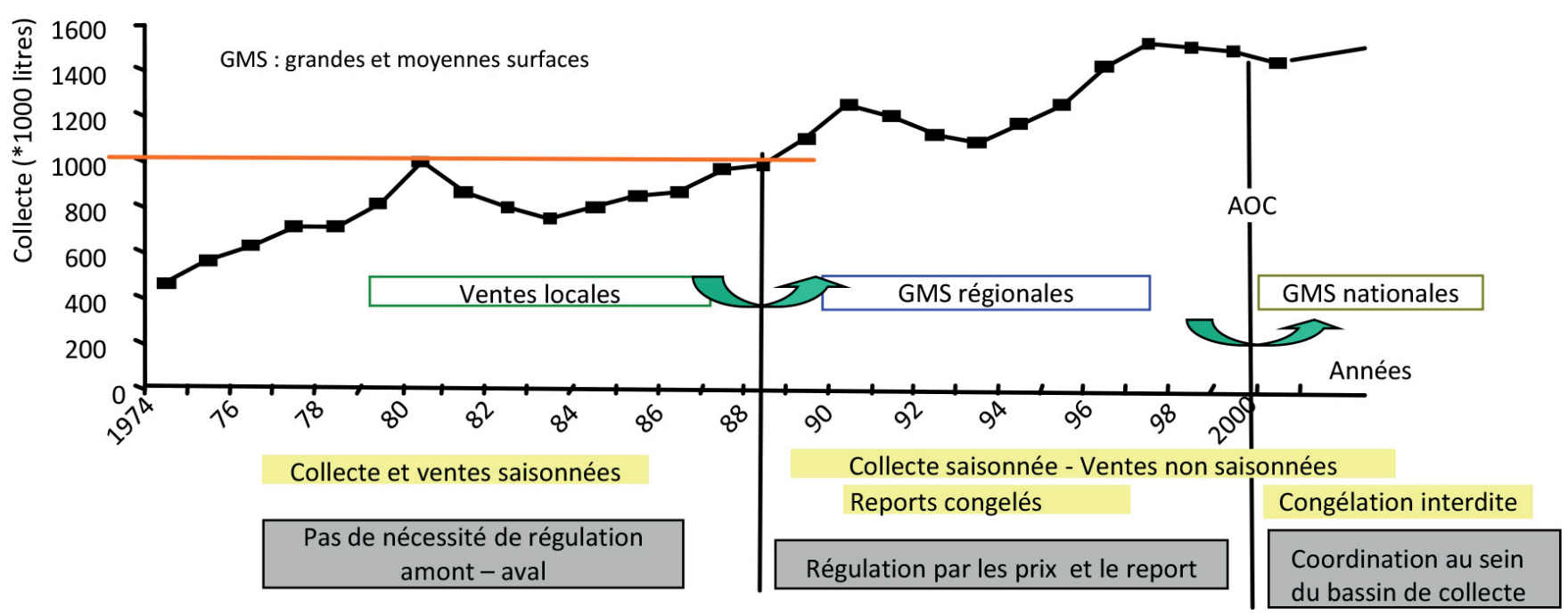

Figure 3 : trajectoire de la coopérative laitière caprine cévenole entre 1974 et 2006.

collecte et de la stratégie commerciale sur le temps long. Cette représentation factuelle et chronologique a été mise en discussion avec l'ensemble des acteurs. Les gestionnaires-coop se sont appuyés sur cette schématisation pour expliciter l'évolution de la stratégie commerciale de la coopérative au cours de la trajectoire, compte tenu de l'évolution des marchés et de la collecte. Cet exercice a débouché sur l'énonciation en commun de la trajectoire de la coopérative depuis 1974 en trois périodes (figure 3). Durant la première (1974-88), la coopérative collectait et vendait à proximité. La production, la collecte et les ventes étaient saisonnées. La seconde (1988-2000) a été marquée par l'augmentation des volumes collectés (« le seuil du million de litres a été dépassé ») conduisant la coopérative à explorer les marchés éloignés en grande distribution (marchés non saisonnés). «On a mis en place des reports congelés, une grille de prix, on a incité les éleveurs à des mises bas d'automne ». Le modèle de contre-saison était qualifié de «bon pour la coopérative », en opposition aux modèles de saison. La troisième période a débuté avec la mise en place de l'AOC. Pour distinguer leur fromage d'un produit industriel, les acteurs de l'AOC (devenue AOP [P = partagée] en 2001) ont interdit, dans le cahier des charges l'utilisation de reports congelés. Ce choix les obligeait dès lors à repenser leurs moyens de gestion de la saisonnalité de la collecte.

Pour approfondir la réflexion et analyser les conséquences de ce choix sur l'ajustement entre la collecte et les ventes en cours de campagne, nous avons mobilisé ensuite une représentation de la collecte mensuelle en regard des ventes AOP mensuelles. Elle a mis en évidence une évolution de la période creuse vers l'été, comme dans le cas de la coopérative drômoise. Ce constat a été capital pour l'analyse de la situation et des perspectives d'action. Cette représentation graphique a conduit les acteurs à reformuler la question à traiter qui est passée de « comment faire pour augmenter le lait d'automne ?» à « comment faire pour augmenter la collecte entre juillet et décembre ?». Avec cette reformulation, les gestionnaires actaient qu'un modèle unique calé sur des mises bas d'automne ne permettrait pas à lui seul de résoudre les difficultés de gestion de la saisonnalité sur une période aussi longue (six mois). En revanche, une combinaison de livraisons avec des saisonnalités différentes pourrait être un moyen d'action. La validation collective d'une telle lecture a revalorisé l'intérêt de la diversité des systèmes d'élevage présents et a inspiré des perspectives d'action à travers l'identification de complémentarités entre systèmes d'élevage. La représentation et l'énonciation de la trajectoire, ainsi que la reformulation collective de la question à traiter au regard de la collecte ont été actées dans un écrit partagé avec tous les acteurs.

- Des livraisons à la diversité des systèmes d'élevage et des pratiques Nous (chercheurs) avons représenté la diversité des systèmes d'élevage (figure 4) à partir de trois clés d'entrée (la période de reproduction, le profil de livraison, et le calendrier de conduite de l'alimentation et du pâturage). Nous avons utilisé le même cadre de représentation 
que celui utilisé pour représenter le mode de conduite et ses liens à la production du troupeau de chaque élevage. Cette représentation de la diversité a été mise en débat avec l'ensemble des acteurs. Les éleveurs ont identifié des proximités fonctionnelles entre leurs modes de production. Ils ont collectivement validé la diversité décrite et se sont individuellement situés dans ces modes de fonctionnement. Un nouvel écrit a consigné cette représentation de la diversité des fonctionnements, les logiques sous-jacentes et les positionnements des éleveurs.

\section{Analyser les interactions entre activités}

En compilant les livraisons des éleveurs qui se situaient dans une même stratégie de conduite du troupeau, nous avons représenté la contribution à la collecte de chaque mode de fonctionnement (figure 5). Cette représentation a permis aux acteurs de réfléchir aux rôles spécifiques de chaque type de système d'élevage pour l'approvisionnement de la coopérative sur la période considérée comme cruciale (juillet à décembre). Ils ont ainsi constaté que les systèmes d'élevage ayant des mises bas de printemps (saison naturelle) assuraient $60 \%$ de l'approvisionnement en été et début automne, et que les systèmes dessaisonnés (mises bas en automne) assuraient $60 \%$ de la collecte en automne et début hiver. Ce constat a fait évoluer le regard des acteurs sur l'intérêt de la diversité au sein du bassin de collecte de la laiterie. Les systèmes d'élevage ayant des mises bas en saison naturelle, considérés jusqu'alors comme traditionnels, en opposition aux systèmes de contre-saison qualifiés par les éleveurs de «bons pour la coop », ont été légitimés à nouveau au regard du rôle qu'ils jouaient dans l'approvisionnement de la laiterie en été.

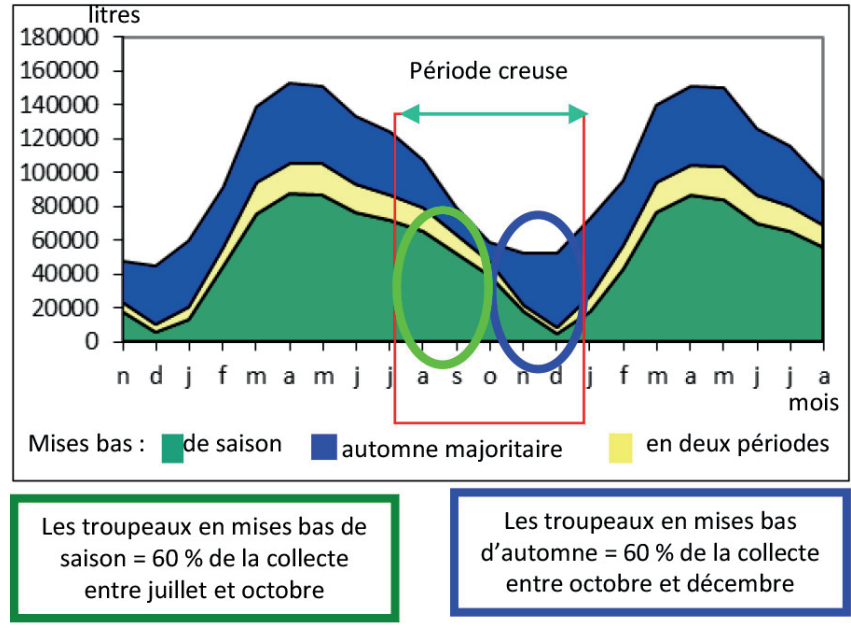

Figure 5 : contribution à la collecte de lait caprin des trois systèmes « idealtypes » d'élevage cévenol.

\section{Penser le futur : identifier les pistes d'action (étape 3)}

Raisonnant les complémentarités entre modèles d'élevage au regard de la collecte, les acteurs ont identifié trois « idéaltypes » (Weber, 1956 ; Schnapper, 1999) de systèmes d'élevage (tableau I) : faire du

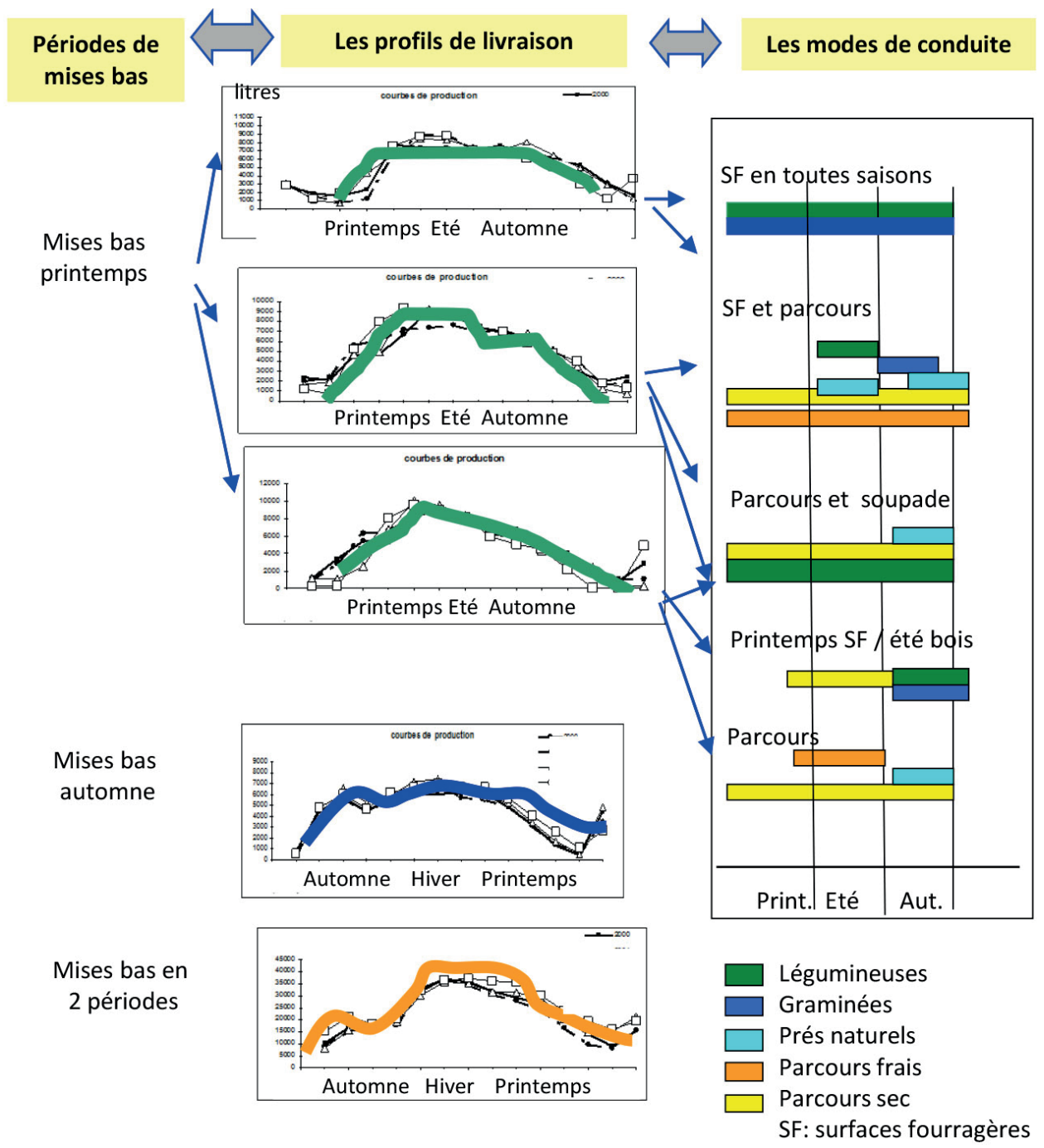

Figure 4 : diversité des systèmes d'élevage caprin cévenol en trois clés d'entrée (extraits). 
lait à l'herbe et tenir le lait jusqu'en été, faire du lait d'hiver et démarrer la production en septembre-octobre, et produire en toute saison. Chaque éleveur s'est situé dans l'idéaltype le plus proche de son projet d'élevage. Suite à ce positionnement, nous avons travaillé avec chacun sur les modifications techniques et organisationnelles qu'il jugeait réalisables au cours de l'année à venir pour contribuer au mieux à la collecte sur le créneau identifié. Pour les éleveurs en mise bas de printemps, la réflexion a porté principalement sur la gestion du pâturage et l'identification de périodes critiques pour la ressource (fin de printemps et été). Pour ceux en mise bas d'automne, la question a porté principalement sur la constitution de stock et l'équilibre foin/pâture sur les parcelles cultivées. Enfin les éleveurs pratiquant deux périodes de mise bas étaient plus confrontés à la difficulté de gérer dans le temps l'équilibre des lots de reproduction. L'exercice a débouché sur la simulation d'une courbe de production prévisionnelle par élevage. Ceci a été acté dans un écrit. Une partie des éleveurs a utilisé cet écrit sur la campagne suivante pour comparer le prévu et le réalisé, et pour ajuster chemin faisant certaines pratiques. Après compilation des prévisions de livraison discutées avec chaque éleveur, nous avons simulé la répartition de la collecte de la laiterie en année $n+1$.

Tout au long de ce dispositif, divers types de représentations graphiques ont été utilisés et transformés. Le tableau II montre les rôles spécifiques de ces représentations dans le processus de conception.

\section{DISCUSSION}

\section{Objets factuels, temporels et graphiques à l'échelle des entités de gestion}

L'intérêt ici était d'utiliser des objets simples, factuels, situés au niveau des entités de gestion pour favoriser l'implication des éleveurs ou des gestionnaires dans la réflexion (Napoléone et Soulard, 2010). Ainsi, à la vue de la courbe de lait de leur troupeau ou de la collecte, ils replongeaient dans l'action de tous les jours, dans leurs difficultés qu'ils mettaient en mots dans leurs récits. Utiliser la production du troupeau ou encore le lait livré, c'était mobiliser les mêmes repères que ceux de l'éleveur ou du gestionnaire. Ce type d'objet facilitait leur investigation empirique, au cours d'allers-retours entre la modélisation et l'action de tous les jours.
Issus des pratiques, ces objets factuels se distinguaient d'indicateurs résultant de calculs techniques ou économiques, par exemple les résultats du contrôle laitier qui renvoyaient à la performance et à la productivité et orientaient l'analyse sur une évaluation experte. Les indicateurs technico-économiques auraient présenté le risque de mettre à distance l'éleveur du fait des positions et représentations sociales, comme le montre Darré (1994 ; 2006). Avec de tels indicateurs, nous n'aurions pas pu analyser la situation en mettant au centre l'action et le sens qu'elle avait pour les acteurs.

\section{Faciliter la prise de recul sur ses pratiques}

Le premier type de support graphique (le lait troupeau ou la collecte) mettait l'acteur dans une posture exploratoire au regard de ses propres activités. Il facilitait l'expression du récit de vie sur le déroulement de l'activité. Ce récit permettait de repérer les « objets de l'action» auxquels l'éleveur faisait référence, pour coconstruire petit à petit une seconde génération de graphiques représentant le déroulement de l'activité (la conduite du troupeau et l'utilisation du territoire, ou une chronique pour la coopérative mettant en lien ventes, stocks, collectes, ou autre). Cette nouvelle représentation graphique constituait un support à partir duquel l'acteur formulait le mode d'organisation et de gestion de l'activité. Ce cheminement réflexif débouchait sur une modélisation du mode d'organisation (par exemple sous forme de séquences, de périodes de changement, d'éléments structurels dans chaque période, de facteurs de changement et de leviers d'action). Cette première partie de la démarche concernait les activités élémentaires et pouvait être conduite avec des éleveurs comme avec des gestionnaires de coopérative.

\section{Se situer par rapport aux autres}

La représentation de la diversité des pratiques et des systèmes d'élevage a été construite en conservant le cadre de formalisation des activités élémentaires. Garder un même cadre pour passer de l'exploitation à la diversité des systèmes d'élevage a aidé chaque éleveur à se situer par rapport aux autres et à identifier des proximités fonctionnelles. Cette représentation a permis aux acteurs de partager une lecture en commun de la diversité des fonctionnements des systèmes d'élevage et de repérer la façon dont chacun se situait dans cette diversité. D’autre part, la représentation choisie au plus près des pratiques

\section{Tableau I}

Des systèmes d'élevage cévenol «idealtypes » aux ajustements de pratiques individuels

\section{Idéaltype}

\section{Du lait à l'herbe}

Une mise bas unique en saison (février-mars) pour " faire du lait à l'herbe » et avoir une période de repos (des animaux et des éleveurs) au cours du tarissement. "J'ai besoin d'arrêter de produire, et de renouveler l'envie avec l'arrivée du printemps et les ventres des chèvres qui s'arrondissent. »

\section{Du lait en hiver}

Une mise bas unique en automne pour faire du lait d'hiver et avoir une période de repos l'été. "Cela me permet de faire les foins plus facilement et d'être disponible l'été avec la famille. »

\section{Du lait en toute saison}

Pour régulariser la production sur l'année le travail sur l'élevage et la trésorerie

\section{Enjeux au regard de la collecte \\ Les moyens d'action}

Tenir le lait jusqu'en juillet août

Démarrer la production dès septembre octobre

Livrer du lait en toute période
Le principal levier est la gestion du pâturage. Par ex. des petites parcelles sont mises en culture pour éviter des chutes de production à des périodes très précises comme le passage printemps été. Ou bien des légumineuses sont implantées pour compléter en été le pâturage sur parcours.

La gestion la reproduction est pensée pour réussir la reproduction à contre-saison.

Un des enjeux principaux est la qualité et la quantité des stocks de fourrage pour réussir la première partie de la lactation.

Arriver à gérer des lots de mises bas et à maintenir l'équilibre entre les lots au fil des années. Certains animaux peuvent être conduits en lactation longue (taris tous les deux ans). 


\section{Tableau II}

Retour sur les représentations graphiques (nature et caractéristiques) de la coordination entre les éleveurs et une coopérative laitière caprine cévenole

\begin{tabular}{|c|c|c|c|}
\hline Type d'objet & Contenu des graphiques & $\begin{array}{l}\text { Rôles dans le processus } \\
\text { de conception collective }\end{array}$ & $\begin{array}{l}\text { Acteurs impliqués } \\
\text { dans l'échange }\end{array}$ \\
\hline Courbe de livraison & Livraisons quotidiennes & Aide à la formalisation & \\
\hline $\begin{array}{l}\text { Calendrier des } \\
\text { pratiques d'élevage }\end{array}$ & $\begin{array}{l}\text { Livraisons quotidiennes } \\
\text { Reproduction } \\
\text { Pâturages utilisés } \\
\text { Alimentation en chèvrerie } \\
\text { Travail }\end{array}$ & $\begin{array}{l}\text { Aide à la prise de recul } \\
\text { Parler de ce que l'on fait }\end{array}$ & L'éleveur \\
\hline \multirow[t]{2}{*}{$\begin{array}{l}\text { Représentation } \\
\text { de la diversité des } \\
\text { modes d'élevage }\end{array}$} & $\begin{array}{l}\text { Profils des livraisons } \\
\text { Organisation reproduction } \\
\text { Organisation du pâturage, } \\
\text { de l'alimentation en chèvrerie }\end{array}$ & $\begin{array}{l}\text { Identification de proximité } \\
\text { Connaissances sur la diversité } \\
\text { Construction d'une lecture commune }\end{array}$ & Entre éleveurs \\
\hline & & $\begin{array}{l}\text { Ouvrir le champ des possibles } \\
\text { Elargir les points de vue }\end{array}$ & $\begin{array}{l}\text { Avec les gestionnaires } \\
\text { de la laiterie }\end{array}$ \\
\hline \multirow[t]{2}{*}{$\begin{array}{l}\text { Courbe de collecte } \\
\text { et chronique } \\
\text { de la coopérative }\end{array}$} & $\begin{array}{l}\text { La collecte quotidienne en année } \\
\text { n-1 } \\
\text { Volumes annuels depuis } 1974\end{array}$ & $\begin{array}{l}\text { Aide à la formalisation } \\
\text { et à la prise de recul }\end{array}$ & \multirow[b]{2}{*}{$\begin{array}{l}\text { Gestionnaires } \\
\text { de laiterie }\end{array}$} \\
\hline & $\begin{array}{l}\text { Volumes annuels collectés } \\
\text { Lieux de ventes } \\
\text { Evolution de la demande } \\
\text { Produits fabriqués }\end{array}$ & Parler de ce que I'on fait & \\
\hline $\begin{array}{l}\text { Trajectoire } \\
\text { de la coopérative }\end{array}$ & $\begin{array}{l}\text { Volumes annuels collectés } \\
\text { Lieux de ventes } \\
\text { Evolution de la demande } \\
\text { Produits fabriqués } \\
\text { Evénements particuliers } \\
\text { Outils de coordination }\end{array}$ & $\begin{array}{l}\text { Aide à l'échange } \\
\text { Aide à l'interprétation et au diagnostic } \\
\text { Construction d'une lecture commune } \\
\text { Ouverture du champ des possibles } \\
\text { (reformulation de la question) }\end{array}$ & Entre gestionnaires \\
\hline $\begin{array}{l}\text { Contribution } \\
\text { à la collecte }\end{array}$ & $\begin{array}{l}\text { La collecte quotidienne } \\
\text { en année n-1 } \\
\text { Le cumul des livraisons } \\
\text { quotidiennes par idéaltype }\end{array}$ & $\begin{array}{l}\text { Aide au diagnostic } \\
\text { Pistes d'action } \\
\text { Conception collective } \\
\text { Aide aux coordinations }\end{array}$ & $\begin{array}{l}\text { Eleveurs et } \\
\text { gestionnaires }\end{array}$ \\
\hline
\end{tabular}

a favorisé l'échange entre éleveurs sur les liens entre leurs pratiques et les profils de production troupeau (soit de livraison). Cet exercice a fait émerger des savoirs d'action des éleveurs. Il a contribué à l'émergence de connaissances situées et partagées, directement utilisables pour la suite par les éleveurs (élaboration d'un prévisionnel, réalisation, comparaison entre le prévisionnel et le réalisé, analyses des écarts et réflexions sur les adaptations à réaliser, etc.).

\section{Objets de l'action passeurs de frontières}

Ces objets permettaient aussi d'établir des liens fonctionnels entre les activités présentes (la gestion des 27 élevages et celle de la collectetransformation-commercialisation). Chaque acteur avait sa propre interprétation de la situation, compte tenu de ses activités et de ses objectifs. Ce type d'outil permettait à chacun de rendre lisibles les éléments qu'il utilisait pour construire son interprétation. Un échange sur ces clés respectives de lecture pouvait s'engager. Il s'agissait ici d'une négociation entre les acteurs pour déboucher sur une lecture et un diagnostic partagé de la situation.

La formulation par écrit de cette interprétation concertée et les questions à traiter qui en résultaient étaient des étapes cruciales qui conditionnaient les pistes d'action envisagées. Nous rejoignons ici le concept d'objet-frontière (Star et Griesemer, 1989) qui permet à des personnes ayant des rationalités différentes de travailler ensemble. Pour Star (2010), ces « objets-frontière sont à la fois temporels, encastrés dans l'action, sujets à réflexion et à l'adaptation locale ». Henderson (1991) insiste sur l'intérêt des représentations visuelles pour faciliter les interprétations croisées entre différents groupes professionnels au cours du processus cognitif. Elles facilitent l'élaboration d'un langage commun et la construction de critères d'appréciation partagés (Torre et Chia, 2001 ; Chia, 2004).

Dans la phase de recherche de solutions, la représentation graphique facilite l'imagination de nouveaux scénarios d'organisation. Scénario est pris ici au sens d'un ensemble formé par la description d'une situation future et par le cheminement des événements - qui doivent présenter une cohérence - permettant de passer de la situation d'origine à une situation future (Godet, 1991). Plusieurs scénarios peuvent être imaginés, représentés, mis en discussion, pour n'en retenir à la fin qu'un seul estimé à la fois souhaitable pour diverses raisons (notamment techniques, économiques, travail) et réalisable aux yeux des acteurs. 


\section{Intérêt des objets intermédiaires construits à partir des pratiques dans la mise en place d'actions collectives}

Les représentations graphiques utilisées dans cette étude ont permis la prise de recul et facilité l'analyse conjointe entre acteurs concernés par la gestion de la collecte, considérée comme une ressource commune. La démarche rejoint celle mise en place dans d'autres travaux de coordination d'acteurs autour de la gestion d'une ressource, comme pour les problématiques de gestion du territoire. Ces travaux mobilisent en général des objets intermédiaires pour aider des acteurs ayant des points de vue très différents à se construire une vision partagée de la dynamique de la ressource commune. Nombre d'entre eux utilisent la coconstruction de modélisation de l'état et de la dynamique de la ressource commune (Lardon et al, 2001). Par exemple la démarche de modélisation d'accompagnement (Bousquet et al., 2002) propose des jeux de rôles pour aider les acteurs à se mettre en situation et à imaginer des scénarios d'évolution.

La particularité de la démarche de notre étude était de modéliser dans un premier temps chacune des activités élémentaires, avec chacun des acteurs, avant de travailler avec l'ensemble des acteurs sur la diversité, puis sur les interactions entre activités. Cet exercice a contribué à la prise de recul de chaque acteur sur sa propre situation et l'a aidé à se situer par rapport aux autres et à sa contribution à l'ensemble du résultat. La recherche de scénarios en était ainsi relativement finalisée, permettant des allers-retours entre le niveau collectif et le niveau individuel, ce qui a facilité l'appropriation et la mise en situation par la suite.

Nous avons insisté dans cet article sur le rôle des représentations graphiques bâties à partir des pratiques et des objets techniques, dans l'aide à la conception innovante et dans la coordination. Pour autant, d'autres types d'informations externes à la situation d'action, techniques ou économiques, auraient pu intervenir ponctuellement à certaines étapes de la réflexion. Ainsi, des informations sur des cas types régionaux, l'intérêt de tel aliment, l'évolution des marchés, les soutiens à l'élevage, ou autre, peuvent être mobilisées dans l'étape de diagnostic ou dans la recherche de pistes d'action. Une simulation de l'intérêt d'un nouveau modèle technique par planification de flux peut aussi apporter des éléments complémentaires de réflexion aux acteurs, dans la phase de recherche de solution.

\section{Reproduire ce type de dispositif?}

Cette étude de cas a mis en évidence l'intérêt d'établir un dispositif sur le temps long (plusieurs années) et d'utiliser des objets intermédiaires issus des pratiques des acteurs. La construction progressive des représentations, la reconnaissance des savoirs et des pratiques locales, la prise en compte du vocabulaire et des entités de gestion utilisées au quotidien ont contribué à la réussite de ce type de dispositif en facilitant la confiance entre acteurs, la symétrie de l'information, l'apprentissage organisationnel.

L'établissement d'une confiance entre acteurs pour construire des objets intermédiaires issus de leurs pratiques ne va pas forcément de soi. Ce processus peut notamment se heurter aux positions sociales des acteurs et aux catégories déjà établies par les acteurs entre les systèmes d'élevage jugés pertinents au regard de la question à traiter (par exemple « les systèmes bons pour la coop »). La négociation de cette question et de la manière de la traiter contribue à l'établissement des conditions de cette confiance. Dans le processus, par tâtonnement, l'animateur propose des objets intermédiaires qui peu à peu vont conduire les acteurs à porter des regards nouveaux sur la situation et à les énoncer. Progressivement, la place de chacun se précise, facilitant la suite du processus de coordination ; à charge de l'animateur d'acter et de mettre en partage ces évolutions.

La symétrie des informations entre acteurs, c'est-à-dire la prise en compte des mêmes informations pour étudier une question, permet à tous d'avoir un regard sur la situation et le problème commun à traiter. Il ne s'agit pas bien sûr de partager toutes les informations utiles à chacun dans la conduite de ses activités. Il s'agit de la symétrie des informations traitées dans le cadre de l'action collective impliquant tous les acteurs. Les représentations graphiques, comme les échanges sur leurs interprétations par les acteurs, contribuent à la construction d'une vision en commun de la situation. Par ailleurs, les acteurs doivent pouvoir mesurer, au fil du processus, l'avancée de la réflexion collective. C'est le rôle joué par les écrits, actant étape après étape les acquis, les connaissances et les questions restant en suspens.

L'apprentissage organisationnel peut être vu à divers niveaux, de l'élevage à la coopérative, à la relation éleveurs - gestionnaires. Les discussions sur la diversité des modèles techniques, ou encore sur leurs intérêts au regard d'une courbe de collecte contribuent à l'identification de règles, de principes, de critères d'appréciation.

Les principes présentés dans ce texte et sous-tendant le dispositif mis en place ont été testés dans plusieurs situations françaises (Napoléone, 2001, Napoléone et Chia, 2010) et à ce titre présentent une certaine généricité. Toutefois, ce dispositif n'est pas une démarche clé en main. Il doit être adapté à chaque situation pour trouver localement la bonne façon de mettre en place le cheminement d'aide à la coordination et la forme la plus pertinente à donner aux objets intermédiaires, ainsi que le montre Etienne (2012) pour les supports de géoprospective.

\section{CONCLUSION}

A la question posée en introduction « La diversité des systèmes d'élevage présents sur le bassin de collecte peut-elle devenir un moyen d'action pour réguler la collecte au cours de l'année ? », nous pouvons répondre oui, mais... Oui, la diversité des systèmes de production, permet l'activation de complémentarités pour mieux ajuster la saisonnalité de la collecte laitière sur un bassin de collecte à celle des ventes. Mais cela nécessite d'avoir une lecture partagée de la situation pour imaginer des pistes d'action coordonnées entre le management à l'échelle des élevages et celui concernant la gestion de la collecte, de la transformation et des ventes.

Pour aider à cette coordination nous avons montré l'intérêt de construire des objets intermédiaires au plus près des pratiques pour aider à la prise de recul et à l'intercompréhension. Nous avons montré aussi l'importance de mettre en place un dispositif facilitant les échanges entre acteurs. Ce processus aide, dans un premier temps, à déconstruire le schéma initial que chaque acteur a de la situation, schémas qui peuvent comporter des césures entre eux, que ce soit entre la production et la transformation, ou entre des façons différentes de concevoir ce qui est un modèle de production. Par la suite, le dispositif facilite une reconstruction collective du cadre d'interprétation de la situation. Dans ce processus, les objets intermédiaires pragmatiques et la construction de récits prennent une place centrale. Ils aident petit à petit à mettre en synergie des points de vue et à imaginer des perspectives d'action négociées en cohérence avec des enjeux individuels et collectifs.

\section{REFERENCES}

Bousquet F., Bareteau O., d'Aquino P., Etienne M., Boissau S., Aubert S., Le Page C., et al., 2002. Multi-agent systems and role games: Collective learning processes for ecosystem management. In: Complexity and ecosystem management: The theory and practice of multi-agent systems (Ed. Janssen Marco A.). Edward Elgar, Cheltenham, UK, 248-285

Chia E., 2004. Principles, methods of participatory research: proposal for draft animal power. Rev. Elev. Med. Vet. Pays Trop., 57 (3-4): 233-240, doi : 10.19182/remvt.9895

Daré W., Ducrot R., Botta A., Etienne M., 2009. Repères méthodologiques pour la mise en œuvre d'une démarche de modélisation d'accompagnement Lirac projet ComMod. Cardère, Avignon, France, 128 p. 
Darré J.P., 1994. Pairs et experts dans I'agriculture. Dialogues et production de connaissances pour l'action. Erès, Ramonville Saint-Agne, France, 228 p.

Darré J.-P., 2006. La recherche coactive de solutions entre agents de développement et agriculteurs. GRET, 112 p. (coll. Etudes et travaux)

Etienne M., 2011. Companion modelling: an adaptative approach? In: Companion modelling: a participatory approach to support sustainable development (Ed. Etienne M.). Quae, Versailles, France, 271-278 (coll. Update Sciences and Technologies)

Etienne M., 2012, La modélisation d'accompagnement : une forme particulière de géoprospective. Espace Géogr., 41 : 128-137, doi: 10.3917/eg.412.0128

Godet M., 1991. Méthode des scénarios. In : De l'anticipation à I'action. Dunod, Paris, France, 39-55

Henderson K., 1991. Flexible sketches and inflexible data bases: visual communication, conscription devices, and boundary objects in design engineering. Sci. Technol. Hum. Values, 16 (4): 448-473, doi: 10.1177/016224399101600402

Lardon S., Maurel P., Piveteau V., 2001. Représentations spatiales et développement territorial. Hermès Sciences, Paris, France, 438 p.

Lorino P., Nefussi J., 2007. Tertiarisation des filières et reconstruction du sens à travers des récits collectifs. Rev. Fr. Gest. (170) : 75-92, doi : 10.3166/ rfg.170.75-92

Napoléone M., 2001. De la gestion de la répartition de la production laitière d'un troupeau à la gestion des approvisionnements d'une firme. Options Méditerr. Sér. A (46) : 177-181

Napoléone M., 2008, Comment les systèmes d'élevage caprin répondent-ils à l'évolution des besoins d'une coopérative laitière ? In : L'élevage en mouvement - flexibilité et adaptation des exploitations d'herbivores. Quae, Versailles, France, 219-228
Napoléone M., Chia E., 2010. Repenser la coordination entre agriculteur et coopérative laitière - Vers une gestion concertée de la saisonnalité de la collecte. Gérer Comprendre (102) : 58-69, doi: 10.3917/geco.102.0058

Napoléone M., Lasseur J., Etienne M., 2008. Devices based on models to accompany stakeholders in enhancing collective learning and action on livestock farming systems. In: 8th Symp. IFSA, Clermont-Ferrand, France, 6-10 July 2008

Napoléone M., Soulard C., 2010. From agricultural practices to boundaries' objects in the collective actions design. In: Proc. Symp. Innovation and Sustainable Development in Agriculture and Food, Montpellier, France, 28-30 June 2010, 11 p.

Schnapper D., 1999. La compréhension sociologique. Démarche de l'analyse typologique. PUF, Paris, France, $125 \mathrm{p}$.

Star S., 2010. Ceci n'est pas un objet-frontière : Réflexions sur l'origine d'un concept. Rev. Anthropol. Connaiss., 4 (1) : 18-35, doi: 10.3917/rac.009.0018

Star S.L., Griesemer J.R., 1989. Translations and boundary objects: amateurs and professionals in Berkeley's Museum of Vertebrate Zoology, 1907-39. Soc. Stud. Sci., 19 (3): 387-420, doi : 10.1177/030631289019003001

Torre A., Chia E., 2001. Pilotage d'une AOC fondée sur la confiance. Gérer Comprendre (65) : 55-67

Vinck D., 1999. Ingénieur au quotidien. Ethnographie de l'activité de conception et d'innovation. PUG, Fontaine, France, $232 \mathrm{p}$.

Weber M., 1956. Essai sur la théorie de la science. Publié à titre posthume. Traduit de I'allemand par Chavy J.P., Dampierre E., 1971. Plon, Paris, France, 411 p.

\section{Summary}

Napoléone $\mathbf{M}$. Intermediate objects to help coordination between breeders and dairies. Example of a goat dairy cooperative in Southern France

Managing the seasonality of milk production is a challenge for collection and processing companies. We explored here the relevance of enhancing complementarities between production systems in a collection basin. The implementation of a coordination mechanism was proposed to identify these complementarities with regard to an issue negotiated between the farmers and the dairy. It was designed as an itinerary: i) identify primary activities, ii) analyze their interactions, iii) phrase a shared diagnosis, and iv) identify courses of action. At each step, we proposed to produce graphic representations built from the practices of the actors and the objects to which they related. These representations were used as intermediate objects. From an action-research project conducted with a small dairy cooperative in the south of France, we showed that the representations of deliveries or collection could be used as intermediate objects to enable the understanding of herd management or marketing strategy of the cooperative, and to help analyze the interactions between these activities and the development of a common project. In conclusion, simple and pragmatic representations have been shown to be effective and interesting tools to adopt in the course of collective actions involving coordination between breeders and cooperatives.

Keywords: goats, milk collection, dairy cooperatives, seasonality, pastoralists, operations research, Mediterranean zone

\section{Resumen}

Napoléone M. Objetos intermedios para ayudar a la coordinación entre criadores y lecherías. El ejemplo de una cooperativa de productos lácteos caprinos en el sur de Francia

La gestión de la estacionalidad de la producción de leche es un desafío para las empresas de recolección y procesamiento. Exploramos aquí el interés de valorar las complementariedades entre los sistemas de producción presentes en una cuenca de recolección. Se propuso el establecimiento de un mecanismo de coordinación para identificar estas complementariedades alrededor de un tema negociado entre los criadores y la lechería. Se concibió como un itinerario: a) la formalización de las actividades elementales, b) el análisis de sus interacciones, c) la formulación de un diagnóstico concertado, y d) la identificación de vías de acción. En cada etapa, se propusieron representaciones gráficas basadas en las prácticas de los actores y los objetos sobre las cuáles éstos se basaron. Estas representaciones se utilizaron como objetos intermedios. Sobre la base de un proyecto de investigación de acción llevado a cabo con una pequeña cooperativa de productos lácteos en el sur de Francia, demostramos que las representaciones de la distribución o recolección podían utilizarse como objetos intermedios para facilitar la comprensión del manejo de hato y la estrategia comercial de la cooperativa, facilitando el análisis de las interacciones entre estas actividades y el desarrollo de un proyecto conjunto. En conclusión, las representaciones simples y pragmáticas demostraron ser herramientas efectivas e interesantes a utilizar en el contexto de acciones colectivas que involucran la coordinación entre criadores y cooperativas.

Palabras clave: caprino, recogida de leche, cooperativas lecheras, estacionalidad, pastoralistas, investigación operativa, zona mediterránea 
